\title{
SOLUTION TO A CLASS OF INTEGRAL EQUATIONS WITH AN APPLICATION TO MAGNETOHYDRODYNAMICS
}

\author{
R. D. Gibson and P. H. Roberts
}

Dedicated to the memory of Charles Lange.

ABstract. The singular integral equation,

$$
\int_{\eta}^{1} \frac{a\left(\eta_{1}\right) d \eta_{1}}{\left(\eta_{1}-\eta\right)^{\nu}}=\int_{-1}^{\eta} \frac{a\left(\eta_{2}\right) d \eta_{2}}{\left(\eta-\eta_{2}\right)^{\nu}}+f(\eta), \quad-1 \leq \eta \leq 1, \quad 0<\nu<1,
$$

is solved in the homogeneous case $(f=0)$ by a simple transformation and in the inhomogeneous case by reducing it to a Riemann-Hilbert equation. The results simplify a solution first obtained by Hunt and Stewartson for the flow of electrically conducting fluid along a rectangular duct in the limit of large externally applied magnetic field.

\section{Introduction}

This paper solves the singular integral equation

$$
\int_{\eta}^{1} \frac{a\left(\eta_{1}\right) d \eta_{1}}{\left(\eta_{1}-\eta\right)^{\nu}}=\int_{-1}^{\eta} \frac{a\left(\eta_{2}\right) d \eta_{2}}{\left(\eta-\eta_{2}\right)^{\nu}}+f(\eta), \quad-1 \leq \eta \leq 1,
$$

where $a$ is to be determined from given $f$, and $\nu$ is a constant $(0<\nu<1)$. The application that prompted the study of (1.1) is described in $\S 4$. In this section, we apply a transformation that leads to a reformulation of (1.1) as a functional equation for $a$, and to a simple solution of that equation in the homogeneous case $(f=0)$, for which

$$
\int_{\eta}^{1} \frac{a\left(\eta_{1}\right) d \eta_{1}}{\left(\eta_{1}-\eta\right)^{\nu}}=\int_{-1}^{\eta} \frac{a\left(\eta_{2}\right) d \eta_{2}}{\left(\eta-\eta_{2}\right)^{\nu}}, \quad-1 \leq \eta \leq 1
$$

In $\S 2$ we form a bridge to the better-studied Riemann-Hilbert problem [7] in the special case $^{1}$

$$
A(\zeta) \cot \frac{1}{2} \pi \nu-\frac{1}{\pi} f_{-1}^{1} \frac{A(\eta) d \eta}{\eta-\zeta}=F(\zeta), \quad-1 \leq \zeta \leq 1,
$$

where $A$ is to be determined from given $F$. This link enables us to solve (1.1) directly, even for $f \neq 0$. In $\S 3$, we briefly examine generalized forms of (1.1).

Received May 4, 1994, revised August 31, 1994.

1991 Mathematics Subject Classification: 45G05, 76W05.

Key words and phrases: singular integral equations, magnetohydrodynamics, duct flow.

${ }^{1}$ The horizontal line through the integral sign signifies that the principal part is taken. 
We first define $g(\zeta)$ by

$$
g(\zeta)=\frac{\sin \pi \nu}{\pi} \int_{-1}^{\zeta}-\frac{f(\eta) d \eta}{(\zeta-\eta)^{1-\nu}}
$$

Solving this Abel equation for $f$, we have

$$
f(\eta)=\int_{-1}^{\eta} \frac{g^{\prime}\left(\eta_{2}\right) d \eta_{2}}{\left(\eta-\eta_{2}\right)^{\nu}}, \quad-1 \leq \eta \leq 1,
$$

so that (1.1) may be written as

$$
\int_{\eta}^{1} \frac{a\left(\eta_{1}\right) d \eta_{1}}{\left(\eta_{1}-\eta\right)^{\nu}}=\int_{-1}^{\eta} \frac{\left[a\left(\eta_{2}\right)+g^{\prime}\left(\eta_{2}\right)\right] d \eta_{2}}{\left(\eta-\eta_{: 2}\right)^{\nu}}, \quad-1 \leq \eta \leq 1 .
$$

We may project these integrals onto the same interval $0 \leq u \leq 1$ of $u$ through the transformations

$$
\eta_{1}=\frac{(1+\eta)-(1-\eta) u}{(1+\eta)+(1-\eta) u}, \quad \eta_{2}:=\frac{(1+\eta) u-(1-\eta)}{(1+\eta) u+(1-\eta)} .
$$

In this way we obtain

$$
\int_{0}^{1} \frac{H\left(\eta_{1}, \eta_{2}\right) d u}{u^{1-\frac{1}{2} \nu}(1-u)^{\nu}}=0
$$

where

$$
H\left(\eta_{1}, \eta_{2}\right)=\left(1-\eta_{1}^{2}\right)^{1-\frac{1}{2} \nu} a\left(\eta_{1}\right)-\left(1-\eta_{2}^{2}\right)^{1-\frac{1}{2} \nu}\left[a\left(\eta_{2}\right)+g^{\prime}\left(\eta_{2}\right)\right] .
$$

It follows from (1.8) that

$$
H(\eta, u)=u^{1-\frac{1}{2} \nu}(1-u)^{\nu} \frac{\partial G(\eta, u)}{\partial u}
$$

where $G(\eta, u)$ is a function satisfying

$$
G(\eta, 0)=G(\eta, 1)
$$

It is, however, far from being an arbitrary function since, according to (1.9), $G$ must be such that $H$, considered as a function of $\eta_{1}$ and $\eta_{2}$, is separable:

$$
H\left(\eta_{1}, \eta_{2}\right)=M\left(\eta_{1}\right)-N\left(\eta_{2}\right)
$$

The homogeneous equation (1.2) clearly admits the simple solution $G=0$, and (1.9) and (1.10) then demand that

$$
\left(1-\eta_{1}^{2}\right)^{1-\frac{1}{2} \nu} a\left(\eta_{1}\right)=\left(1--\eta_{2}^{2}\right)^{1-\frac{1}{2} \nu} a\left(\eta_{2}\right),
$$

for all $\eta_{1}$ and $\eta_{2}\left(\leq \eta_{1}\right)$. It follows that, for so:ne constant $C$,

$$
a(\eta)=C\left(1-\eta^{2}\right)^{\frac{1}{2} \nu-1} .
$$

Our first conjecture is that this is the only admissible solution of (1.2). Our second conjecture is that, when $N(\eta)-M(\eta) \equiv\left(1-\eta^{2}\right) g^{\prime}(\eta)$ is given, there is only one choice of $G$ for which (1.10)-(1.12) hold. The solution to (1.1) is then unique apart from the addition of the general solution (1.14) of (1.2). These two conjectures are the result of solving (1.1) by determining $A$ from (1.3), as we now do. 


\section{Link to Riemann-Hilbert problem}

Let us define $\alpha(\zeta)$ by

$$
\alpha^{\prime}(\zeta)=a(\zeta), \quad \alpha(1)=0
$$

Multiply (1.1) by $(\zeta-\eta)^{1-\nu} d \eta$ and integrate from $\eta=-1$ to $\eta=\zeta$. We then obtain, using also (1.4),

$$
I \equiv \int_{-1}^{\zeta} \frac{d \eta}{(\zeta-\eta)^{1-\nu}} \int_{\eta}^{1} \frac{\alpha^{\prime}\left(\eta_{1}\right) d \eta_{1}}{\left(\eta_{1}-\eta\right)^{\nu}}=\frac{\pi}{\sin \pi \nu}[\alpha(\zeta)-\alpha(-1)+g(\zeta)]
$$

Reversing the order of integration on the left-hand side of (2.2), we find that

$$
I=\lim _{\epsilon \rightarrow+0}\left[\int_{-1}^{\zeta-\epsilon} \alpha^{\prime}\left(\eta_{1}\right) J\left(\eta_{1}, \zeta, \nu\right) d \eta_{1}+\int_{\zeta+\epsilon}^{1} \alpha^{\prime}\left(\eta_{1}\right) J\left(\zeta, \eta_{1}, 1-\nu\right) d \eta_{1}\right]
$$

where

$$
J\left(\eta_{1}, \zeta, \nu\right)=\int_{-1}^{\eta_{1}} \frac{d \eta}{\left(\eta_{1}-\eta\right)^{\nu}(\zeta-\eta)^{1-\nu}}
$$

On making the substitution

$$
\eta=\zeta-\frac{\left(\zeta-\eta_{1}\right)}{t}
$$

we see that

$$
J\left(\eta_{1}, \zeta, \nu\right)=\int_{T}^{1} \frac{d t}{t(1-t)^{\nu}}
$$

where

$$
T=\frac{\zeta-\eta_{1}}{1+\zeta}
$$

Integrating by parts in (2.3), we now obtain

$$
\begin{aligned}
I=\lim _{\epsilon \rightarrow+0} & {\left[\alpha(\zeta-\epsilon) \int_{\epsilon /(1+\zeta)}^{1} \frac{d t}{t(1-t)^{\nu}}-\alpha(\zeta+\epsilon) \int_{\epsilon /(1+\zeta)}^{1} \frac{d t}{t(1-t)^{1-\nu}}\right.} \\
& \left.+(1+\zeta)^{\nu} \int_{-1}^{\zeta-\epsilon} \frac{\alpha\left(\eta_{1}\right) d \eta_{1}}{\left(\eta_{1}-\zeta\right)\left(1+\eta_{1}\right)^{\nu}}+(1+\zeta)^{\nu} \int_{\zeta+\epsilon}^{1} \frac{\alpha\left(\eta_{1}\right) d \eta_{1}}{\left(\eta_{1}-\zeta\right)\left(1+\eta_{1}\right)^{\nu}}\right]
\end{aligned}
$$

It may easily be shown that

$$
\int_{\delta}^{1} \frac{d t}{t(1-t)^{\nu}} \sim-\ln \delta-\frac{\Gamma^{\prime}(1-\nu)}{\Gamma(1-\nu)}+\Gamma^{\prime}(1), \quad \delta \rightarrow+0
$$


It follows that

$$
\begin{gathered}
\lim _{\epsilon \rightarrow+0}\left[\alpha(\zeta-\epsilon) \int_{\epsilon /(1+\zeta)}^{1} \frac{d t}{t(1-t)^{\nu}}-\alpha(\zeta+\epsilon) \int_{\epsilon /(1+\zeta)}^{1} \frac{d t}{t(1-t)^{1-\nu}}\right] \\
\quad=\alpha(\zeta)\left[\frac{\Gamma^{\prime}(\nu)}{\Gamma(\nu)}-\frac{\Gamma^{\prime}(1-\nu)}{\Gamma(1-\nu)}\right]=\alpha(\zeta) \frac{d}{d \nu} \ln [\Gamma(\nu) \Gamma(1-\nu)] \\
\quad=\alpha(\zeta) \frac{d}{d \nu} \ln \left[\frac{\pi}{\sin \pi \nu}\right]=-\pi \alpha\left(\iota_{\zeta}\right) \cot \pi \nu
\end{gathered}
$$

and that

$$
I=-\pi \alpha(\zeta) \cot \pi \nu+(1+\zeta)^{\nu} f_{--1}^{1} \frac{\alpha\left(\eta_{1}\right) d \eta_{1}}{\left(\eta_{1}-\zeta\right)\left(1+\eta_{1}\right)^{\nu}}
$$

Equation (2.2) takes the form

$$
\alpha(\zeta) \cot \frac{1}{2} \pi \nu+[g(\zeta)-\alpha(-1)] \operatorname{cosec} \pi \nu-\frac{(1+\zeta)^{\nu}}{\pi} f_{-1}^{1} \frac{\alpha\left(\eta_{1}\right) d \eta_{1}}{\left(\eta_{1}-\zeta\right)\left(1+\eta_{1}\right)^{\nu}}=0
$$

To cast this in the form (1.3), we now replace $\alpha$ by $A$, where

$$
\alpha(\zeta)=(1+\zeta)^{\nu} A(\zeta), \quad \gamma=\lim _{\zeta \rightarrow-1}(1+\zeta)^{\nu} A(\zeta)
$$

Then (2.12) reduces to (1.3) with

$$
F(\zeta)=\frac{\gamma-g(\zeta)}{(1+\zeta)^{\nu}} \operatorname{cosec} \pi \nu
$$

Standard theory (e.g., see [7] or p.183 of [6]) provides a solution of (1.3) as

$$
\begin{aligned}
A(\zeta)=\frac{1}{2} & F(\zeta) \sin \pi \nu+\frac{\sin ^{2} \frac{1}{2} \pi \nu}{\pi}\left(\frac{1-\zeta}{1+\zeta}\right)^{\frac{1}{2} \nu}\left(\frac{1+\eta}{1-\eta}\right)^{\frac{1}{2} \nu} \frac{F(\eta) d \eta}{(\eta-\zeta)} \\
& +\frac{K(1-\zeta)^{\frac{1}{2} \nu}}{(1+\zeta)^{1+\frac{1}{2} \nu}}
\end{aligned}
$$

where $K$ is an arbitrary constant, representing the solution of the homogeneous form of (1.3).

We now use (2.15) to solve (1.1). We first observe that the $K$ term in (2.15) would lead to a divergence in (1.1) at $\eta_{2}=-1$ and must therefore be eliminated. From (2.13)-(2.15), we obtain

$$
\alpha(\zeta)=\frac{1}{2}[\alpha(-1)-g(\zeta)]+\frac{\tan \frac{1}{2} \pi \nu}{2 \pi}\left(1-\zeta^{-2}\right)^{\frac{1}{2} \nu} f_{-1}^{1} \frac{[\alpha(-1)-g(\eta)] d \eta}{\left(1-\eta^{2}\right)^{\frac{1}{2}} \nu(\eta-\zeta)}
$$

The integral in (2.16) diverges at $\zeta= \pm 1$. By again using (2.9), we can show that (2.16) becomes an identity in the limit $\zeta \rightarrow-1$; also $(2.1)_{2}$ is confirmed in the limit $\zeta \rightarrow 1$. The term in (2.16) proportional to $\alpha(-1)$ corresponds to the general solution of (1.2). According to $(2.1)_{2}$, this is proportiona. to

$$
\begin{aligned}
a(\zeta) & =\frac{d}{d \zeta}\left[\left(1-\zeta^{2}\right)^{\frac{1}{2} \nu} f_{-1}^{1} \frac{d \eta}{\left(1-\eta^{2}\right)^{\frac{1}{2} \nu}(\eta-\zeta)}\right] \\
& =-\frac{\pi \Gamma(\nu) \cot \frac{1}{2} \pi \nu}{2^{\nu-2}\left[\Gamma\left(\frac{1}{4}\right)\right]^{2}}\left(1-\zeta^{2}\right)^{\frac{1}{2} \nu-1}
\end{aligned}
$$


which also relates this to the more convenient form (1.14).

We may now use (2.16) to write the general solution of (1.1) as

$$
\begin{aligned}
a(\zeta)=- & \frac{1}{2} \frac{d}{d \zeta}\left[g(\zeta)+\frac{\tan \frac{1}{2} \pi \nu}{\pi}\left(1-\zeta^{2}\right)^{\frac{1}{2} \nu} f_{-1}^{1} \frac{g(\eta) d \eta}{\left(1-\eta^{2}\right)^{\frac{1}{2} \nu}(\eta-\zeta)}\right] \\
& +C\left(1-\zeta^{2}\right)^{\frac{1}{2} \nu-1}
\end{aligned}
$$

where $g$ is defined by (1.4). To clarify the symmetries of this solution, we observe that, by multiplying (1.1) by $(\eta-\zeta)^{1-\nu} d \eta$, integrating from $\eta=\zeta$ to $\eta=1$, and repeating the analysis of this section, we obtain

$$
\begin{aligned}
a(\zeta)= & \frac{1}{2} \frac{d}{d \zeta}\left[\widetilde{g}(\zeta)-\frac{\tan \frac{1}{2} \pi \nu}{\pi}\left(1-\zeta^{2}\right)^{\frac{1}{2} \nu} f_{-1}^{1} \frac{\widetilde{g}(\eta) d \eta}{\left(1-\eta^{2}\right)^{\frac{1}{2}} \nu(\eta-\zeta)}\right] \\
& +C\left(1-\zeta^{2}\right)^{\frac{1}{2} \nu-1}
\end{aligned}
$$

where

$$
\widetilde{g}(\zeta)=\frac{\sin \pi \nu}{\pi} \int_{\eta}^{1} \frac{f(\eta) d \eta}{(\eta-\zeta)^{1-\nu}}
$$

If $f$ is symmetric $[f(-\zeta)=f(\zeta)]$, then $\widetilde{g}(-\zeta)=g(\zeta)$ by (1.4) and (2.20). It then follows from (2.18) and (2.19) that $a$ is symmetric. Similarly antisymmetric $f$ leads to antisymmetric $a$; this solution does not involve $C$ and is therefore unique.

\section{A generalization}

Let $h(\eta)$ be a monotonic increasing function in $-1<\eta<1$ that is odd in $\eta: h(-\eta)=$ $-h(\eta)$. Consider the problem of finding $a(\eta)$ such that

$$
\int_{\eta}^{1} \frac{a\left(\eta_{1}\right) d \eta_{1}}{\left[h\left(\eta_{1}\right)-h(\eta)\right]^{\nu}}=\int_{-1}^{\eta} \frac{a\left(\eta_{2}\right) d \eta_{2}}{\left[h(\eta)-h\left(\eta_{2}\right)\right]^{\nu}}+f(\eta), \quad-1 \leq \eta \leq 1 .
$$

We redefine $g$ as

$$
g(\zeta)=\frac{\sin \nu \pi}{\pi} \int_{-1}^{\zeta} \frac{h^{\prime}(\eta) f(\eta) d \eta}{[h(\zeta)-h(\eta)]^{1-\nu}} .
$$

Solving this Abel equation for $f$, we have [11]

$$
f(\eta)=\int_{-1}^{\eta} \frac{g^{\prime}\left(\eta_{2}\right) d \eta_{2}}{\left[h(\eta)-h\left(\eta_{2}\right)\right]^{\nu}}, \quad-1 \leq \eta \leq 1
$$

so that (3.1) may be written as

$$
\int_{\eta}^{1} \frac{a\left(\eta_{1}\right) d \eta_{1}}{\left[h\left(\eta_{1}\right)-h(\eta)\right]^{\nu}}=\int_{-1}^{\eta} \frac{\left[a\left(\eta_{2}\right)+g^{\prime}\left(\eta_{2}\right)\right] d \eta_{2}}{\left[h(\eta)-h\left(\eta_{2}\right)\right]^{\nu}}, \quad-1 \leq \eta \leq 1
$$

The transformations replacing (1.7) are

$$
\begin{aligned}
\frac{h\left(\eta_{1}\right)}{h(1)} & =\frac{[h(1)+h(\eta)]-[h(1)-h(\eta)] u}{[h(1)+h(\eta)]-[h(1)-h(\eta)] u} \\
\frac{h\left(\eta_{2}\right)}{h(1)} & =\frac{[h(1)+h(\eta)] u-[h(1)-h(\eta)]}{[h(1)+h(\eta)] u-[h(1)-h(\eta)]}
\end{aligned}
$$


Equation (1.8) follows with a redefined $H$ :

$$
\begin{aligned}
H\left(\eta_{1}, \eta_{2}\right)= & \frac{\left[h^{2}(1)-h^{2}\left(\eta_{1}\right)\right]^{1-\frac{1}{2} \nu}}{h^{\prime}\left(\eta_{1}\right)}-a\left(\eta_{1}\right) \\
& -\frac{\left[h^{2}(1)-h^{2}\left(\eta_{2}\right)\right]^{1-\frac{1}{2} \nu}}{h^{\prime}\left(\eta_{2}\right)}\left[a\left(\eta_{2}\right)+g^{\prime}\left(\eta_{2}\right)\right] .
\end{aligned}
$$

The remainder of $\S 1$ follows similar lines, (1.14) being replaced by

$$
a(\eta)=C h^{\prime}(\eta)\left[h^{2}(1)-h^{2}(\eta)\right]^{\frac{1}{2} \nu-1} .
$$

We may also derive the analogue of the Riema:nn-Hilbert problem (1.3). We redefine $A(\zeta)$ and $\gamma$ by

$$
\alpha(\zeta)=[h(1)+h(\zeta)]^{\nu} A(\zeta), \quad \gamma=\lim _{\zeta \rightarrow-1}[h(1)+h(\zeta)]^{\nu} A(\zeta) ;
$$

definition (2.14) for $F$ is unchanged with this $\gamma$. Following mutatis mutandis the reasoning of $\S 2$, we obtain in place of $(1.3)$

$$
A(\zeta) \cot \frac{1}{2} \pi \nu-\frac{1}{\pi} f_{-1}^{1} \frac{A(\eta) d \eta}{h(\eta)-h(\zeta)}=F(\zeta), \quad-1 \leq \zeta \leq 1 .
$$

As an example $\left[h(\eta)=\sin \frac{1}{2} \pi \eta\right]$, we note that the solution (3.7) of the homogeneous equation

$$
\int_{u}^{\frac{1}{2} \pi} \frac{a\left(u_{1}\right) d u_{1}}{\left(\sin u_{1}-\sin u\right)^{\nu}}=\int_{-\frac{1}{2} \pi}^{u} \frac{a\left(u_{2}\right) d u_{2}}{\left(\sin u-\sin u_{2}\right)^{\nu}}
$$

is

$$
a(u)=\bar{C} \sec ^{1-\nu} u,
$$

where $\bar{C}$ is an arbitrary constant.

\section{Magnetohydrodynamic duct flow}

A classic problem of magnetohydrodynamics is to determine the steady flow of liquid metal along a uniform rectilinear duct $(-l<x<l,-1<y<1,-\infty<z<\infty)$ in the presence of a uniform applied magnetic field of unit strength in the $y$-direction, the flow $v$ being driven by a unit adverse pressure gradient in the $z$-direction, i.e., along the duct. In these scaled units, the problem reduces to that of solving

$$
\begin{aligned}
& \frac{\partial^{2} b}{\partial x^{2}}+\frac{\partial^{2} b}{\partial y^{2}}+M \frac{\partial v}{\partial y}=0 \\
& \frac{\partial^{2} v}{\partial x^{2}}+\frac{\partial^{2} v}{\partial y^{2}}+M \frac{\partial b}{\partial y}=-1
\end{aligned}
$$

where $b$, the magnetic field created by the flow, also has only a $z$-component. Solutions to (4.1) and (4.2) are required to satisfy conditions on the walls of the duct, namely

$$
\begin{gathered}
v(x, 1)=v(x,-1)=v(l, y)=v(-l, y)=0 \\
\frac{\partial b}{\partial x}=\mp D_{V} b, \quad \text { on } x= \pm l ; \quad \frac{\partial b}{\partial y}=\mp D_{H} b, \quad \text { on } y= \pm 1 .
\end{gathered}
$$


Conditions (4.3) are the no-slip conditions on the fluid velocity, and (4.4) are the so-called "thin-wall boundary conditions", which are now commonly applied to the magnetic field and which were first introduced by Shercliff [10]. Since the scaled conductance, $D_{V}(\geq 0)$, of each "vertical" wall is the same, the fields $v$ and $b$ are symmetric in $x$; since the scaled conductance, $D_{H}(\geq 0)$, of each "horizontal" wall is the same, $v$ is symmetric in $y$ while $b$ is antisymmetric. One by-product of the solution that is of particular practical interest is the (scaled) mass flux down the duct:

$$
Q=\int_{-l}^{l} d x \int_{-1}^{1} v(x, y) d y .
$$

The literature devoted to the solution of the problem just defined is extensive, and we cite here only a few of the pertinent papers. A proof that the solution of (4.1)-(4.4) is unique was provided by Hunt [4]. In early work, Shercliff [9] solved (4.1)-(4.4) in the case $D_{V}=D_{H}=\infty$ of walls that are perfect electrical insulators, and Chang and Lundgren [1] and Uflyand [15] did likewise for the case $D_{V}=D_{H}=0$ of walls that are perfect electrical conductors. Hunt [3] obtained the solutions for $D_{H}=0$ with $D_{V}$ arbitrary, and also for $D_{V}=\infty$ with $D_{H}$ arbitrary. All these investigations made use of eigenfunction expansions, but this approach is impractical in the case of greatest practical interest,

$$
D_{H}=\infty, \quad D_{V} \text { arbitrary. }
$$

Hunt and Stewartson showed, however, in a celebrated paper [5], how the $D_{V}=0$ case of (4.6) could be solved asymptotically in the limit ${ }^{2}$ of large Hartmann number, $M$. It is significant for the usefulness of such analyses that large values of $M$ can be attained in duct flow experiments in the laboratory and that $Q$ can be measured accurately.

The asymptotic solution of (4.1)-(4.4) involves matching regions of five types:

(1) The core, defined as the region asymptotically far from all walls;

(2) Two Hartmann layers, of thickness $\delta_{H}=M^{-1}$, adjacent to each horizontal wall, but asymptotically far from the vertical walls;

(3) Two Shercliff layers, of thickness $\delta_{S}=M^{-1 / 2}$, adjacent to each vertical wall, but asymptotically far from the horizontal walls;

(4) Four further Hartmann layers, adjacent to the horizontal walls but lying within the Shercliff layers and asymptotically far from the corners;

(5) Four corner regions each lying within an $\mathrm{O}\left(M^{-1}\right)$ distance from one of the corners $x= \pm l, y= \pm 1$.

The situation is sketched in Figure 1. The complete solution is

$$
v=\sum_{n=1}^{5} v_{n}, \quad b=\sum_{n=1}^{5} b_{n}
$$

where $v_{n}$ and $b_{n}$ refer to region $n ; Q$ can be written similarly. Simplifications arise when boundary conditions are applied. In particular, only $v_{1}+v_{2}$ and $b_{1}+b_{2}$ are required to satisfy (4.3) and (4.4) in regions 2, since the remaining parts of $v$ and $b$ are exponentially small; similarly, only $v_{1}+v_{3}$ and $b_{1}+b_{3}$ are required in regions 3 , and only $v_{1}+v_{3}+v_{4}$ and $b_{1}+b_{3}+b_{4}$ in regions 4 . The full solution is needed for

\footnotetext{
${ }^{2}$ We should perhaps remark for clarity that, of the two large parameters mentioned, $D_{H}$ is set infinite before the limit $M \rightarrow \infty$ is considered; see also [13, 14].
} 


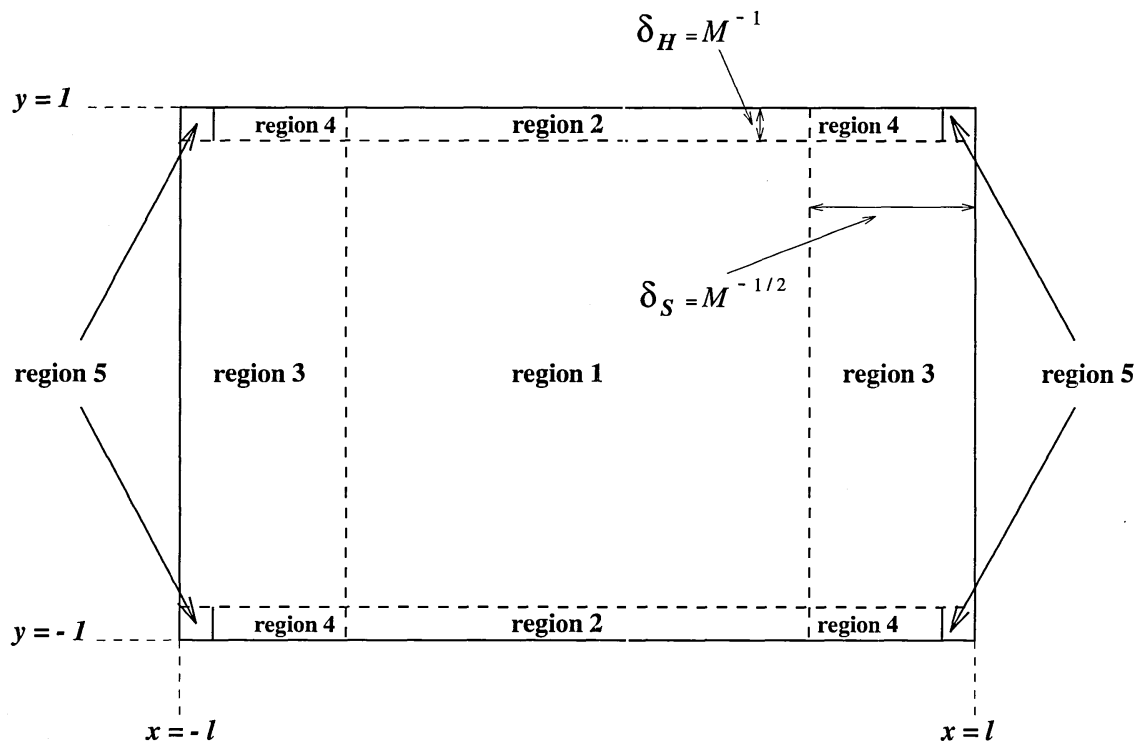

FIGURE 1. Schematic representation of asymptotic regions in magnetohydrodynamics duct flow.

regions 5 and this can only be obtained by solving the full equations (4.1) and (4.2). This problem will not be considered here, and consequently not even the dominant $\mathrm{O}\left(M^{-5 / 2}\right)$ part of $Q_{5}$ is derived. Only an expression for $Q$ accurate to order $M^{-3 / 2}$ will be sought. We restrict attention to case (4.6)

4.1. The core. Assume that $\partial / \partial x$ and $\partial / \partial y$ are $\mathrm{O}(1)$. Bearing in mind the symmetry of the solution, we see that, (4.1) and (4.2) give to leading order

$$
v_{1}=f(x) / M, \quad b_{1}=-y / M,
$$

where $f$ is to be determined by matching (see below). Solution (4.8) can readily be taken to higher order by regular perturbation, e.g.,

$$
v_{1}=\frac{f(x)}{M}+\frac{g(x)}{M^{2}}+\mathrm{O}\left(\frac{1}{M^{3}}\right), \quad b_{1}=-y\left[\frac{1}{M}+\frac{f^{\prime \prime}(x)}{M^{2}}+\mathrm{O}\left(\frac{1}{M^{3}}\right)\right] .
$$

The contribution made by the core to $Q$ dominates all others; by (4.5) and (4.9), it is

$$
Q_{1}=4 \int_{0}^{l}\left[\frac{f(x)}{M}+\frac{g(x)}{M^{2}}\right] d x+\mathrm{O}\left(\frac{1}{M^{3}}\right) .
$$

4.2. The Hartmann layer at $y=1$ far from $x= \pm l$. Assume that $\partial / \partial x$ and $\partial / \partial \eta$ are $\mathrm{O}(1)$, where $\eta=(1-y) / \delta_{H}$. To leading order, (4.1) and (4.2) give

$$
v_{2} \pm b_{2}=F_{ \pm} e^{ \pm \eta}
$$

The requirement that the solution vanishes as $\eta \rightarrow \infty$ requires that $F_{+}=0$, so that

$$
\begin{aligned}
& v_{2}(x, \eta)+b_{2}(x, \eta)=0, \\
& v_{2}(x, \eta)-b_{2}(x, \eta)=2 v_{2}(x, 0) e^{-\eta} .
\end{aligned}
$$


Again, this solution may be taken to higher order by regular perturbation:

$$
v_{2}(x, \eta)=-b_{2}(x, \eta)=\left[v_{2}(x, 0)-\frac{\eta}{M} v_{2}^{\prime \prime}(x, 0) \eta+\cdots\right] e^{-\eta}
$$

The fact that (4.12) holds in particular at $\eta=0$, i.e., $y=1$, is really significant, as it provides the means for removing the uncertainties, $f, g, \ldots$ of the core solution [12]. Since $v_{1}+v_{2}$ and $b_{1}+b_{2}$ must obey (4.3) and (4.4), we find

$$
f(x)=1, \quad g(x)=0,
$$

and (4.9), (4.14) and (4.10) become

$$
\begin{gathered}
v_{1}=\frac{1}{M}+\mathrm{O}\left(\frac{1}{M^{3}}\right), \quad b_{1}=-y\left[\frac{1}{M}+\mathrm{O}\left(\frac{1}{M^{3}}\right)\right] \\
v_{2}(x, \eta)=-b_{2}(x, \eta)=-\left[\frac{1}{M}+\mathrm{O}\left(\frac{1}{M^{3}}\right)\right] e^{-\eta} \\
Q_{1}=\frac{4 l}{M}+\mathrm{O}\left(\frac{1}{M^{3}}\right) .
\end{gathered}
$$

It also follows from (4.17) that

$$
Q_{2}=-4 l \delta_{H} \int_{0}^{\infty} e^{-\eta} d \eta+\mathrm{O}\left(\frac{1}{M^{4}}\right)=-\frac{4 l}{M^{2}}+\mathrm{O}\left(\frac{1}{M^{4}}\right) .
$$

4.3. The Shercliff layer at $x=l$ far from the corners $y= \pm 1$. Assume that $\partial / \partial \xi$ and $\partial / \partial y$ are $\mathrm{O}(1)$, where $\xi=(l-x) / \delta_{S}$. To leading order, (4.1) and (4.2) are

$$
\frac{\partial^{2} b_{3}}{\partial \xi^{2}}+\frac{\partial v_{3}}{\partial y}=0, \quad \frac{\partial^{2} v_{3}}{\partial \xi^{2}}+\frac{\partial b_{3}}{\partial y}=0
$$

Since $v_{1}+v_{3}$ and $b_{1}+b_{3}$ obey (4.3) and (4.4), solutions to (4.20) must satisfy

$$
v_{3}(0, y)=-\frac{1}{M}, \quad b_{3}(0, y)=\frac{y}{M}+\frac{M^{1 / 2}}{D_{V}}\left(\frac{\partial b_{3}}{\partial \xi}\right)_{\xi=0} .
$$

Since $\delta_{H} \ll \delta_{S}$, it is again legitimate to apply (4.12) in regions 4 , in the forms

$$
v_{3}(\xi, 1)+b_{3}(\xi, 1)=0, \quad v_{3}(\xi,-1)-b_{3}(\xi,-1)=0
$$

the latter of which follows from an analysis of the Hartmann layer on $\eta=-1$.

Let

$$
b_{3}(0, y)=\alpha(y) / M
$$

and introduce

$$
X=v_{3}+b_{3} .
$$

Then (4.20)-(4.24) require that

$$
\frac{\partial^{2} X}{\partial \xi^{2}}+\frac{\partial X}{\partial y}=0
$$

where $X$ must obey

$$
X(\xi, 1)=0, \quad X(0, y)=\frac{\alpha(y)-1}{M}
$$


and

$$
\frac{\partial}{\partial \xi}[X(\xi, y)-X(\xi,-y)]=\frac{2 D_{V}}{M^{1 / 2}}[\alpha(y)-y], \quad \text { on } \xi=0 .
$$

The symmetries of the problem require that

$$
\alpha(-y)=-\alpha(y)
$$

so that (4.26) demands that

$$
\alpha(1)=1, \quad \alpha(-1)=-1 .
$$

Equation (4.25) is the heat equation with $1-y$ playing the role of time. It may be solved subject to (4.27) by standard methods, e.g., Laplace transformation. It is found that

$$
X(\xi, y)=\frac{\xi}{2 \pi^{1 / 2} M} \int_{y}^{1} \frac{\left[\alpha\left(\eta_{1}\right)-1\right.}{\left(\eta_{1}-y\right)^{3 / 2}} \exp \left[-\frac{\xi^{2}}{4\left(\eta_{1}-y\right)}\right] d \eta_{1} .
$$

According to (4.27) and (4.30), $\alpha(y)$ must satisfy the integral equation [12]

$$
\int_{y}^{1} \frac{\alpha^{\prime}\left(\eta_{1}\right) d \eta_{1}}{\left(\eta_{1}-y\right)^{1 / 2}}=\int_{-1}^{y} \frac{\alpha^{\prime}\left(\eta_{2}\right) d \eta_{2}}{\left(y-\eta_{2}\right)^{1 / 2}}+2 D_{V}\left(\frac{\pi}{M}\right)^{1 / 2}[\alpha(y)-y] .
$$

From the appropriate solution of (4.31), the leading order of the flux deficit, $-Q_{3}$, due to the Shercliff layers may be computed from (4:5):

$$
\begin{aligned}
Q_{3} & =2 \delta_{S} \int_{-l}^{l} d y \int_{0}^{\infty} \frac{1}{2}[X(\xi, y)+X(\xi,-y)] d \xi \\
& =2 \delta_{S} \int_{-l}^{l} d y \int_{0}^{\infty} X(\xi, y) d \xi \\
& =-\frac{2}{\sqrt{ }\left(\pi M^{3}\right)} \int_{-l}^{l} d y \int_{y}^{1} \frac{\left[1-\alpha\left(\eta_{1}\right)\right]}{\left(\eta_{1}-y\right)^{1 / 2}} d \eta_{1} \\
& =-\frac{2}{\sqrt{ }\left(\pi M^{3}\right)} \int_{-1}^{1} d \eta_{1}\left[1-\alpha\left(\eta_{1}\right)\right] \int_{-1}^{\eta_{1}} \frac{d y}{\left(\eta_{1}-y\right)^{1 / 2}} \\
& =-\frac{4}{\sqrt{ }\left(\pi M^{3}\right)} \int_{-1}^{1}\left[1-\alpha\left(\eta_{1}\right) \cdot\left(1+\eta_{1}\right)^{1 / 2} d \eta_{1} .\right.
\end{aligned}
$$

Equation (4.31) can be solved explicitly in the two limits $D_{V} / M^{1 / 2}=\infty$ and $D_{V} / M^{1 / 2}=0$. In the former case, $\alpha(y)=y$ and (4.32) gives

$$
Q_{3}=-\frac{128}{15 \sqrt{ }\left(2 \pi \cdot M^{3}\right)}
$$

e.g., see [8]. In the latter case, (4.31) reduces to

$$
\int_{y}^{1} \frac{\alpha^{\prime}\left(\eta_{1}\right) d \eta_{1}}{\left(\eta_{1}-y\right)^{1 / 2}}=\int_{-1}^{y} \frac{\alpha^{\prime}\left(\eta_{2}\right) d \eta_{2}}{\left(y-\eta_{2}\right)^{1 / 2}}
$$

which is the $\nu=\frac{1}{2}$ form of (1.2). From the general solution (1.14), we have

$$
\alpha(\eta)=1-\frac{\sqrt{ }(8 \pi)}{\left[\Gamma\left(\frac{1}{4}\right)\right]^{2}} \int_{\eta}^{1} \frac{d \eta_{1}}{\left(1-\eta_{1}^{2}\right)^{3 / 4}}
$$


where ${ }^{3}$ we have evaluated $C$ by applying (4.29). Substituting this result into (4.32), we obtain

$$
Q_{3}=-\frac{8 \pi}{\left[\Gamma\left(\frac{1}{4}\right)\right]^{2} M^{3 / 2}}
$$

The case $D_{V} / M^{1 / 2}=0$ was first solved by Hunt and Stewartson [5]. They used the transformation described in $\S 2$ to cast (4.34) into Riemann-Hilbert form ${ }^{3}$

$$
A(\zeta)-\frac{1}{\pi} f_{-1}^{1} \frac{A(\eta) d \eta}{\eta-\zeta}=\frac{2}{(1+\zeta)^{1 / 2}}, \quad-1 \leq \zeta \leq 1 .
$$

Appealing to [2], Hunt and Stewartson [5] gave

$$
\begin{aligned}
A(\eta) & =\frac{8(1-\eta)^{1 / 4}}{\pi(1+\eta)^{5 / 4}} \int_{1}^{\infty} \frac{s^{2} d s}{\left(s^{4}-1\right)^{1 / 2}\left(s^{4}+\psi\right)} \\
& =\frac{(1-z)^{1 / 4}}{\pi z^{5 / 4}} \int_{0}^{1} \frac{d u}{u^{1 / 4}(1-u)^{1 / 2}(1-\phi u)} \\
& =\frac{4 \sqrt{ }(2 \pi)}{\left[\Gamma\left(\frac{1}{4}\right)\right]^{2}} \cdot \frac{(1-z)^{1 / 4}}{z^{5 / 4}} F_{1}\left(1, \frac{3}{4} ; \frac{5}{4} ; 1-z^{-1}\right),
\end{aligned}
$$

where $\psi=-\phi=(1-z) / z=(1-\eta) /(1+\eta)$ and ${ }_{2} F_{1}$ denotes the hypergeometric function, here degenerate. Using the transformation properties of these functions, we find that we can rewrite $(4.38) \mathrm{as}^{3}$

$$
\begin{aligned}
A(\eta) & =\frac{4 \sqrt{ }(2 \pi)}{z^{1 / 4}\left[\Gamma\left(\frac{1}{4}\right)\right]^{2}} F_{1}\left(\frac{1}{4}, \frac{3}{4} ; \frac{5}{4} ; z\right) \\
& =\frac{\sqrt{ }(2 \pi)}{z^{1 / 4}\left[\Gamma\left(\frac{1}{4}\right)\right]^{2}} \int_{0}^{1} \frac{d q}{q^{3 / 4}(1-z q)^{3 / 4}} \\
& =\frac{\sqrt{ }(2 \pi)}{z^{1 / 2}\left[\Gamma\left(\frac{1}{4}\right)\right]^{2}} \int_{0}^{z} \frac{d s}{s^{3 / 4}(1-s)^{3 / 4}} \\
& =\frac{\sqrt{ }(8 \pi)}{(1+\eta)^{1 / 2}\left[\Gamma\left(\frac{1}{4}\right)\right]^{2}} \int_{-1}^{\eta} \frac{d t}{\left(1-t^{2}\right)^{3 / 4}} .
\end{aligned}
$$

This agrees with (4.35).

\section{Final remarks}

We have solved (1.1) not only in the homogeneous case (1.2) but also for general $f$. We have transformed (1.1) into a functional equation to which we could readily find the simple solution (1.14) in the homogeneous case. The inhomogeneous case was transformed into a Riemann-Hilbert equation that we solved by standard methods. In our alternative approach to (1.1), we were unable to determine whether, and if so in what circumstances, the functional equation obtained by eliminating $H$ between (1.9)

\footnotetext{
${ }^{3}$ To make $(2.1)_{2}$ consistent with $(4.29)_{1}$, we have taken $\alpha(\S 4)=1-\alpha(\S 2)=1-(1+\zeta)^{1 / 2} A$, so that $a(\S 4)=-a(\S 2)$ and $\gamma=2$ by $(4.29)_{2}$.
} 
and (1.12) possesses more than one solution. Stated another way, let $\eta$ and $u$ in (1.9) and (1.12) be expressed as functions of $\eta_{1}$ and $\eta_{2}$ defined by (1.7), i.e.,

$$
u=\left[\left(\frac{1-\eta_{1}}{1+\eta_{1}}\right)\left(\frac{1+\eta_{2}}{1-\eta_{2}}\right)\right]^{1 / 2}, \quad \frac{1+r_{i}}{1-r_{i}}=\left[\left(\frac{1+\eta_{1}}{1-\eta_{1}}\right)\left(\frac{1+\eta_{2}}{1-\eta_{2}}\right)\right]^{1 / 2} .
$$

Considering $\eta_{1}$ and $\eta_{2}$ as new independent variables and writing $G(\eta, u)=-2 P\left(\eta_{1}, \eta_{2}\right)$, we see that

$$
H\left(\eta_{1}, \eta_{2}\right)=L\left(\eta_{1}, \eta_{2}\right)\left[\left(1-\eta_{1}^{2}, \frac{\partial P}{\partial \eta_{1}}-\left(1-\eta_{2}^{2}\right) \frac{\partial P}{\partial \eta_{2}}\right]\right.
$$

where

$$
L\left(\eta_{1}, \eta_{2}\right)=\left(\left[\left(\frac{1+\eta_{1}}{1-\eta_{1}}\right)\left(\frac{1-\eta_{2}}{1+\eta_{2}}\right)\right]^{1 / 4}-\left[\left(\frac{1-\eta_{1}}{1+\eta_{1}}\right)\left(\frac{1+\eta_{2}}{1-\eta_{2}}\right)\right]^{1 / 4}\right)^{\nu}
$$

and by (1.11)

$$
P(\eta, \eta)=P(1,-1)
$$

By $(1.12), H\left(\eta_{1}, \eta_{2}\right)$ must be of the form $M\left(\eta_{1}\right)-N\left(\eta_{2}\right)$. The unresolved question is whether, in the homogeneous case $M(\eta)=N(\eta)$, there exist $P$ (other than $P=0$ ) for which this is true and whether, in the inhomogeneous case, more than one such $P$ exists. Although this question remains open, our solution via the Riemann-Hilbert equation encourages us to believe that our solıtions are complete. It may be worth remarking that the solution of (1.1) in the cası $\nu=0$ and $f(1)=-f(-1)$ is clearly unique [though trivial: $a(\eta)=\frac{1}{2} f^{\prime}(\eta)$ ].

\section{References}

1. C. C. Chang and T. S. Lundgren, Duct flow in magnetohydrodynamics, Z. angew. Math. Phys. 12 (1961), 100-114.

2. H. K. Cheng and N. Rott, Generalizations of the inversion formula of thin airfoil theory, J. Rat. Mech. Anal. 3 (1954), 357-382.

3. J. C. R. Hunt, Magnetohydrodynamic flow in rectangular ducts, J. Fluid Mech. 21 (1965), 577590 .

4. - A uniqueness theorem for magnetohydrodynamic duct flows, Math. Proc. Cambridge Philos. Soc. 65 (1969), 319-327.

5. J. C. R. Hunt and K. Stewartson, Magnetohydrodynamic flow in rectangular ducts. II, J. Fluid Mech. 23 (1965), 563-581.

6. R. P. Kanwal, Linear Integral Equations, Academic Press, New York, 1971.

7. N. I. Muskhelishvili, Singular Integral Equations: Boundary Problems of Function Theory and their Application to Mathematical Physics, Noordhoff, Groningen, 1953. Reprinted by Dover Publications Inc., New York, 1992.

8. P. H. Roberts, An Introduction to Magnetohydrodynamics, Longmans, Green \& Co., London, 1967.

9. J. A. Shercliff, Steady motion of conducting fluid: in pipes under transverse magnetic fields, Math. Proc. Cambridge Philos. Soc. 49 (1953), 136-144.

10. Mech. 1 (1956), 644-666.

11. R. P. Srivastav, A note on certain integral equations of Abel-type, Proc. Edinburgh Math. Soc. (2) 5 (1963), 271-272.

12. K. Stewartson, On the motion of a non-conducting body through a perfectly conducting fluid, J. Fluid Mech. 8 (1960), 82-96.

13. D. J. Temperley and L. Todd, The effects of wall conductivity in magnetohydrodynamic duct flow at high Hartmann numbers, Math. Proc. Cambridge Philos. Soc. 69 (1971), 337-351. 
14. _ Some remarks on a class of plane, linear boundary problems, J. Inst. Math. Appl. 18 (1976), 309-324.

15. Y. S. Uflyand, Flow stability of a conducting fluid in a rectangular channel in a transverse magnetic field, Sov. Phys. Tech. Phys. 5 (1961), 1191-1193.

The Chancellery, Queensland University of Technology, 2 George Street, Brisbane, QueENSLAND 4001, Australia

Department of Mathematics, University of California, Los Angeles, California 90024, U.S.A. 